\title{
Metabolic syndrome: Pathophysiology and Consequences
}

\author{
Anugya Bharti* and Archana Kushwaha \\ Department of Foods and Nutrition, GB Pant University of Agriculture and Technology, \\ Pantnagar, Uttarakhand-263145, India \\ *Corresponding author
}

\section{Keywords}

Insulin resistance, Abdominal adiposity, Atherogenic dyslipidemia and Endothelial dysfunction

\section{Article Info}

Accepted:

28 August 2020

Available Online:

10 September 2020

\section{A B S T R A C T}

The metabolic syndrome refers to the co-occurrence of or clustering of several known cardiovascular risk factors, including insulin resistance, abdominal adiposity, atherogenic dyslipidemia and endothelial dysfunction. These conditions are interrelated and share underlying mechanisms and pathways. It identifies a subgroup of patients with shared pathophysiology who are at high risk of developing cardiovascular disease, hypertension and type II diabetes. Various definitions for metabolic syndrome were given but NCEP ATP III definition is one of the most widely used criteria of metabolic syndrome. Insulin resistance affects Phosphoinositide 3-kinase (PI3K) pathway whereas Mitogen activated protein (MAP) kinase pathway remains unaffected. This results in hyperglycemia and reduced nitric oxide production leading to endothelial dysfunction. Excess abdominal obesity is associated with a potentially atherogenic lipoprotein profile, hyperinsulinemia, releases of nonesterified fatty acids and reduced levels of adiponectin. All these features of the metabolic syndrome leads to type 2 diabetes mellitus, hypertension, dyslipidemia, and atherosclerotic vascular disease, particularly coronary artery disease and PCOS (Polycystic ovarian syndrome). Sympathetic hyperactivity, leptin resistance, insulin resistance or rennin angiotensin aldosterone system hyperactivation leads to increased anti -natriuretic effect and thus, may contribute towards hypertension in obese individuals. Insulin resistance also leads to PCOS by increasing level of GnRH (Gonadotrophin releasing hormone) and LH (luteinizing hormone), increases ACTH and adrenal cortex stimulation and reducing the level of sex hormone-binding globulin (SHBG). Thus, by considering the central features of the metabolic syndrome and how they are related, we may be able to better understand the pathophysiology that links them with each other and with the increased risk of CVD and other diseases.

\section{Introduction}

The metabolic syndrome refers to the cooccurrence of or clustering of several known cardiovascular risk factors, including hyperglycemia/insulin resistance, obesity and dyslipidemia. It identifies patients who are at high risk of developing atherosclerosis, CVD and type 2 diabetes (T2D) (Halcox \& Quyyumi, 2006). By considering the central features of the metabolic syndrome and how they are related, we may be able to better understand the pathophysiology that links them with each other and with the increased risk of CVD. 
Criteria for Clinical Diagnosis of Metabolic syndrome

According to NCEP ATP III (2005), metabolic syndrome is present if three or more of the following five criteria are met:

Waist circumference over 40 inches (men) or 35 inches (women)

Blood pressure over 130/85 mmHg

Fasting triglyceride (TG) level over 150 $\mathrm{mg} / \mathrm{dl}$

Fasting high-density lipoprotein (HDL) level less than $40 \mathrm{mg} / \mathrm{dl}$ (men) or $50 \mathrm{mg} / \mathrm{dl}$ (women)

Fasting blood sugar over $100 \mathrm{mg} / \mathrm{dl}$ (Huang, 2009).

\section{Features of metabolic syndrome}

\section{The four features of metabolic syndrome includes}

\section{Insulin resistance}

Insulin is produced by the pancreas in response to hyperglycemia and stimulates removal of glucose from the circulation and uptake and use by various tissues of the body such as skeletal muscle, liver and adipose tissue (Huang, 2009). In the skeletal muscle and adipose tissue, insulin stimulates uptake and use of glucose by translocation of the glucose transporter (GLUT4) to the cell surface. In the skeletal muscle and liver, insulin prevents glycogenolysis and stimulates the synthesis of glycogen from glucose. In the liver, insulin also reduces gluconeogenesis and prevents entry of more glucose into the bloodstream. In adipose tissue, insulin inhibits lipolysis, and stimulates glucose uptake. The net effect of all of these changes is to increase glucose uptake, reduce circulating glucose levels and increase the conversion of glucose into the storage molecules either glycogen or fat (Kim et al., 2006).

Insulin resistance typically increases with increasing body fat composition when peripheral tissues such as skeletal muscle, adipose tissue and liver cells do not respond appropriately to insulin. This results in hyperinsulinemia and hypergycaemia and is an important predisposing mechanism for development of the metabolic syndrome and type II diabetes mellitus.

The binding of insulin to the insulin receptor activates tyrosine phosphorylation and results in activation of two parallel pathways: the phosphoinositide 3-kinase (PI3K) pathway and the mitogen activated protein (MAP) kinase pathway. PI3K activates further two pathways: 3-phosphoinositide-dependent protein kinase 1(PDK1) kinase and Akt kinase. Akt kinase stimulates translocation of the insulin responsive glucose transporter GLUT4 to the cell surface in skeletal muscle and adipose tissue, thus, increases the glucose uptake. In vascular endothelial cells, Akt kinase activates endothelial nitric oxide synthase (eNOS) that causes release of nitric oxide (NO). NO has vasodilator and anti inflammatory effects. The MAP kinase pathway mediates endothelin-1 (ET-1) production that leads to vasoconstriction (Huang, 2009).

In insulin resistance, Phosphoinositide 3kinase (PI3K) pathway is affected that reduces GLUT4 translocation resulting in decrease glucose uptake by skeletal muscles and adipose tissue ultimately causes hyperglycaemia. Also, there is reduced nitric oxide production thus leading to Pro inflammatory condition and endothelial dysfunction. By contrast, the MAP kinase pathway is unaffected, so there is continued ET-1 production. In this ways, insulin resistance leads to vascular abnormalities that predispose to atherosclerosis. 


\section{Abdominal adiposity/ Visceral adiposity}

Abdominal or visceral adiposity is strongly associated with metabolic syndrome and risk of CHD. This is characterized by accumulation of excessive visceral adipose tissue. Abdominal adiposity is associated with insulin resistance. Adipose tissue is a rich source of cytokines, including TNF- $\alpha$ and IL6, adiponectin, and resistin, which are collectively referred to as adipocytokines. Functions of TNF- $\alpha$ include modulation of lipid metabolism; reduction of tyrosine kinase activity in insulin receptors, inhibiting insulin signaling and sensitivity; downregulation of glucose transporter proteins; promotion of pancreatic beta-cell dysfunction; and impairment of endothelial function. Adipocytokines contribute to insulin resistance. Accumulation of excessive visceral adipose tissue (in the presence or absence of obesity) is associated with insulin resistance, hyperinsulinemia, and glucose intolerance. Circulating and tissue levels of TNF- $\alpha$ are typically increased in association with hyperinsulinemia in obesity.

In addition, excess abdominal obesity is associated with a potentially atherogenic lipoprotein profile, which includes (1) hypertriglyceridemia; (2) elevated apolipoprotein B levels; (3) an increased proportion of small, dense LDL particles; and (4) reduced HDL cholesterol concentrations. Visceral fat releases nonesterified fatty acids that overload muscle and liver with lipids, enhancing insulin resistance. Levels of adiponectin are reduced in obesity and are associated with reduced insulin sensitivity and an adverse risk factor profile.

\section{Atherogenic dyslipidemia}

The key features of atherogenic dyslipidemia are hypertriglyceridemia, elevated apolipoprotein B levels, an increased proportion of LDL particles and reduced HDL cholesterol concentrations (Semenkovich, 2006).

Insulin resistance leads to atherogenic dyslipidemia. Insulin resistance causes impairment of muscle glucose uptake and oxidation. This accounts for the increased lipolysis and generation of glycerol and nonesterified fatty acid (NEFA). The excess of NEFA flux will then feed to the liver. In the liver, FFAs serve as a substrate for synthesis of TGs, resulting in more VLDL production. Secondly, the major lipoprotein of very-low-density lipoprotein (VLDL) particles is apolipoprotein B (apoB). Generation of nonesterified fatty acid (NEFA) increases the production of apoB leading to increased VLDL production. Thirdly, insulin normally degrades apoB through PI3Kdependent pathways, so insulin resistance directly increases VLDL production. Another aspect is that insulin is responsible for regulating the activity of lipoprotein lipase, resulting in decreases TGs production and VLDL production. When there is insulin resistance, there is increased VLDL production and so, LDL production. Insulin resistance might lead to reduced LDL receptors. Thus, elevated LDL production and reduced LDL receptors may leads to atheroma production (Miccoli et al., 2008).

\section{Endothelial dysfunction}

Endothelial cells line the inner surface of blood vessels and produces vasoactive substances, including NO (nitric oxide), prostacyclin and endothelins. Nitric oxide (NO) is generated by a complex endothelial cell calcium-calmodulin-dependent enzyme known as endothelial nitric oxide synthase (NOS) that converts arginine to citrulline, releasing NO (Pober et al, 2009). NO has a wide range of biological properties such as Vasodialation, anti - inflammatory effect, anti 
- thrombotic effect and protects the blood vessel from injuries and plaques formation thus, prevents atherosclerosis (Hadi et al., 2005).

The NOS enzyme has $\mathrm{N}$ terminus and $\mathrm{C}$ terminal. The NOS $\mathrm{N}$ terminus (monooxygenase domain) binds to tetrahydrobiopterin and heme. L-arginine binds in the enzyme active site near heme, while molecular oxygen is coordinated directly to the ferrous heme iron. The Cterminal domain binds NADPH, FAD, and FMN cofactors (1). The $\mathrm{N}$ - and C-terminal domains are linked by a short sequence that binds calmodulin, an allosteric effector that is essential for full NOS activity. The factors that affect NOS activity includes insufficient arginine, absence of bound calmodulin and hyperglycemia. NOS can produce superoxide when levels of arginine are insufficient. In the absence of bound calmodulin, the transfer of electrons from the reductase domain to the oxygenase domain is impeded and the catalytic activity of NOS is blunted. And lastly, Hyperglycemia may lead to intracellular changes in the redox state resulting in depletion of the cellular NADPH pool.

\section{Consequences of metabolic syndrome}

\section{Diabetes mellitus}

Abdominal obesity (one of the criteria for defining metabolic syndrome) causes insulin resistance that causes impairment of muscle glucose uptake and oxidation. Thus, there is increased blood sugar level and development of diabetes mellitus.

\section{Hypertension}

Abdominal obesity causes hypertension through various mechanisms. Abdominal obesity causes leptin resistance, thus, increases renal sympathetic nerve activity (RSNA) through systemic infusion of leptin which results in increased BP and hypertension. Secondly, abdominal obesity causes sympathetic hyperactivity resulting in increased renal tubular sodium reabsorption through impaired renal-pressure natriuresis.

Abdominal obesity results in activation of renin-angiotensin system (RAS). There is increased production of angiotensin II and angiotensinogen in obese individuals and elevated plasma aldosterone level. Increased angiotensin II inhibits the action of insulin and inhibit PI3K/Akt signaling, resulting in decreased NO production in endothelial cell and increased vasoconstriction. Also, increased aldosterone level increases the reabsorption of sodium, thus, increases the volume of extracellular fluid in the body. In this way, activated RAS increases blood pressure in metabolic syndrome (Yanai et al., 2008).

\section{Dyslipidemia and heart disease}

Insulin resistance leading to hypertriglyceridemia and also the overproduction of VLDL. There is also increase in LDL production, decreased HDL level and decrease in LDL receptors. Nitric oxide synthase (NOS) activity is also altered due to abdominal obesity and insulin resistance ultimately resulting in dyslipidemia and heart disease.

\section{Polycystic ovary syndrome (PCOS)}

Hyperinsulinemia results in increased $\mathrm{GnRH}$ synthesis and secretion by acting on GnRHsecreting cells and subsequently elevates the LH levels. Higher levels of this hormone induce greater androgen synthesis in ovarian theca cells (TC) on continuous basis resulting in hyperandrogenemia that plays a pivotal role in the development and progression of PCOS. The constant growth of follicles, along 
with nonselection of a dominant unit, leads to the hyperstimulation of several of these structures, so, PCOS is also proposed as polyfollicular ovary syndrome and it leads to hormonal imbalances. Insulin also reinforces adrenal glands as an alternate androgen source parallel to ovaries. Hyperinsulinemia also increases adrenal cortex sensitivity to ACTH stimulation, with increased androgen secretion. Also, elevated insulin concentrations have been associated with lower levels of Sex hormone-binding globulin (SHBG), leading to enhanced bioavailability of androgens (Ambiger, 2016).

In conclusion, the metabolic syndrome refers to the co-occurrence of or clustering of several known cardiovascular risk factors, including insulin resistance, abdominal adiposity, atherogenic dyslipidemia and endothelial dysfunction. These conditions are interrelated and share underlying mechanisms and pathways. Various definitions for metabolic syndrome were given but NCEP ATP III definition is one of the most widely used criteria of metabolic syndrome. Insulin resistance affects PI3K pathway whereas Mitogen activated protein (MAP) kinase pathway remains unaffected. This results in hyperglycemia and reduced nitric oxide production leading to endothelial dysfunction. Excess abdominal obesity is associated with a potentially atherogenic lipoprotein profile, hyperinsulinemia, releases of nonesterified fatty acids and reduced levels of adiponectin. All these features of the metabolic syndrome leads to type 2 diabetes mellitus, hypertension, dyslipidemia, and atherosclerotic vascular disease, particularly coronary artery disease and PCOS (Polycystic ovarian syndrome). Insulin resistance also leads to PCOS by increasing level of GnRH (Gonadotrophin releasing hormone) and LH(luteinizing hormone), increases ACTH and adrenal cortex stimulation and reducing the level of sex hormone-binding globulin (SHBG). Thus, by considering the central features of the metabolic syndrome and how they are related, we may be able to better understand the pathophysiology that links them with each other and with the increased risk of CVD and other diseases.

\section{References}

Ambiger, S. (2016). Study of insulin resistance and lipid profile in polycystic ovarian syndrome. Int J Sci Res Publ, 6, $1-5$.

Hadi, H. A., Carr, C. S., \& Al Suwaidi, J. (2005). Endothelial dysfunction: cardiovascular risk factors, therapy, and outcome. Vascular health and risk management, 1(3), 183.

Halcox, J., \& Quyyumi, A. A. (2006). Metabolic syndrome: overview and current guidelines. Clinical review article. Ed: A. Mazair Zafari, MD, PhD, FACC. Tersedia di: www. turner-white. com [Diunduh 29 Desember 2016].

Huang, P. L. (2009). A comprehensive definition for metabolic syndrome. Disease models \& mechanisms, 2(5-6), 231-237.

Kim, J. A., Montagnani, M., Koh, K. K., \& Quon, M. J. (2006). Reciprocal relationships between insulin resistance and endothelial dysfunction: molecular and pathophysiological mechanisms. Circulation, 113(15), 1888-1904.

Miccoli, R., Keyzer, D. D., Giuseppe Penno, D., \& Stefano Del Prato, D. (2008). Insulin resistance and lipid disorders. Future lipidology, 3(6), 651664.

Pober, J. S.; Min, W. and Bradley, J. R. 2009. Mechanisms of endothelial dysfunction, injury, and death. Annual Review of Pathological Mechanical Disease. 4: 71-95.

Semenkovich, C. F. (2006). Insulin resistance 
and atherosclerosis. The Journal of clinical investigation, 116(7), 18131822.

Yanai, H., Tomono, Y., Ito, K., Furutani, N., Yoshida, H., \& Tada, N. (2008). The underlying mechanisms for development of hypertension in the metabolic journal, 7(1), 1-6.

\section{How to cite this article:}

Anugya Bharti and Archana Kushwaha. 2020. Metabolic syndrome: Pathophysiology and Consequences. Int.J.Curr.Microbiol.App.Sci. 9(09): 3723-3728.

doi: https://doi.org/10.20546/ijcmas.2020.909.459 